Harold A. McAlister and William I. Hartkopf (eds.)

\title{
Statistics of Double and Multiple Stars in the Solar Neighborhood ${ }^{1}$
}

\author{
VICTOR V. ORLOV
}

Astronomical Observatory, St. Petersburg University, Bibliotechnaya pl. 2, 19890, St. Petersburg Petrodvorets, Russia

\section{OLEG A. TITOV}

Institute of Applied Astronomy, Russian Academy of Sciences, Zhdanovskaya st. 8, 197042, St. Petersburg, Russia

\begin{abstract}
We study the multiplicity function and physical properties of single, double, and multiple stellar systems in the solar neighbourhood ( $<10 \mathrm{pc}$ ), using a new preliminary machine-readable version of the Gliese \& Jahreiss Catalogue (1991).

The ratio of $(n+1)$-ple to $n$-ple systems is a constant fraction of about $1 / 4$ for $n=1$, $2,3,4$. The luminosity function $f(M)$ for primaries in binaries has an additional peak at $M \in\left(6^{m}, 8^{m}\right)$ that is absent in $f(M)$ for single stars and secondary components. A significant correlation between the absolute magnitudes $M_{1}$ and $M_{2}$ of the components in binaries takes place.
\end{abstract}

\section{INTRODUCTION}

The modern concepts of star formation assume an origin of stars in groups by gravitational fragmentation. Therefore the multiplicity function $f(n)$ may reflect some cosmogonic aspects of the star formation scenaria. Batten \& Fletcher (1989) have estimated the function $f(n)$ using the catalogue of spectroscopic binary systems by Batten et al. (1989). They have shown that the ratio of $(n+1)$-ple to $n$-ple systems is a constant fraction of about $15 \%$. Batten (1973) has found that the theoretical function is

$$
f_{t}(n)=0.582 / n !,
$$

if the multiple stars are formed as condensates around the randomly distributed cores.

In this work we consider the most unbiased sample of 227 stellar systems within $\mathrm{r} \leq 10 \mathrm{pc}$ from the sun.

We discuss the multiplicity function $f(n)$ for this population (Section 2); the luminosity functions as well as the distributions by spectra and color indexes $B-V($ Section 3$)$; and also the correlation between the absolute magnitudes $M_{1}$ and $M_{2}$ of components in the binaries (Section 4).

\section{MULTIPLICITY FUNCTION}

In Table 1 we show the distribution $\mathrm{f}(\mathrm{n})$ for 227 nearby star systems by the multiplicity $\mathrm{n}$, as well as the ratio $\kappa_{n}=f(n+1) / f(n)$. For comparison we present the theoretical multiplicity function (1) and the ratios $\kappa_{t}(n)$ for it.

\footnotetext{
${ }^{1}$ The authors of this paper were unable to attend the Colloquium - eds.
} 

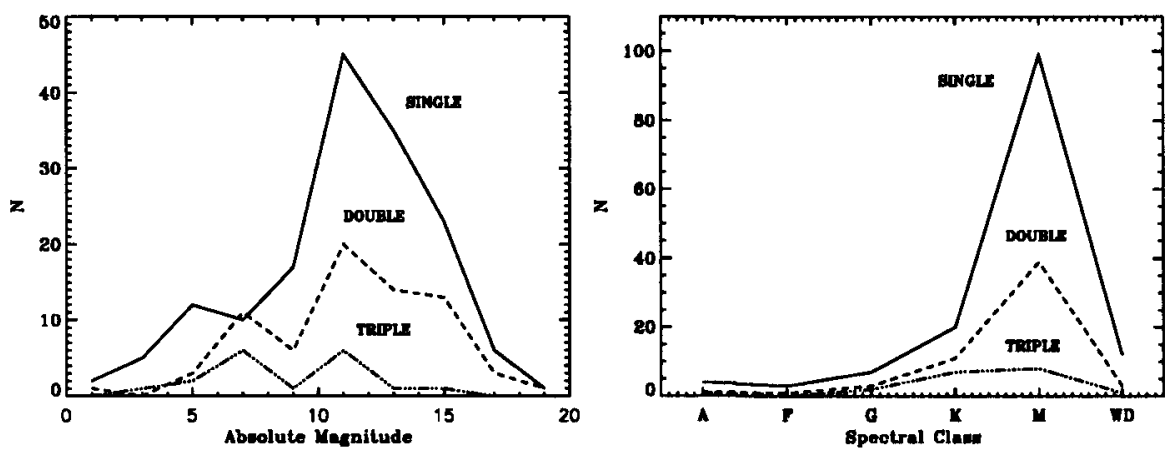

FIGURE 1. (left) The luminosity function $N(M)$ for single, double, and triple star components. (right) The distributions $\mathrm{N}(\mathrm{Sp})$ by spectral type.

We compared these results with the data by Duquennoy \& Mayor (1991) for the solar type primaries (two last columns).

TABLE 1. The multiplicity function $f(n)$ for nearby stars.

\begin{tabular}{|rrllllll|}
\hline $\mathrm{n}$ & \multicolumn{1}{c}{$\mathrm{N}$} & $f(n)$ & $\kappa_{n}$ & $f_{t}(n)$ & $\kappa_{t}$ & $f_{\triangleleft}(n)$ & $\kappa_{\star}$ \\
\hline 1 & 161 & 0.71 & 0.32 & 0.58 & 0.50 & 0.57 & 0.67 \\
2 & 51 & 0.22 & 0.22 & 0.29 & 0.33 & 0.38 & 0.11 \\
3 & 11 & 0.05 & 0.27 & 0.10 & 0.25 & 0.04 & 0.25 \\
4 & 3 & 0.01 & 0.33 & 0.02 & 0.20 & 0.01 & \\
5 & 1 & 0.004 & & 0.005 & 0.17 & & \\
\hline
\end{tabular}

We do not observe any tendency for $\kappa_{n}$ in our sample. This value is approximately constant and does not depend on $n$; its average value $\bar{\kappa}=0.28 \pm 0.03$. This is in disagreement with decreasing $\kappa_{t}(n)$ and with larger variation of $\kappa_{s}(n)$.

\section{DISTRIBUTIONS}

We have constructed the luminosity functions $f(M)$ for the samples of single, double, and triple stars (Figure 1, left). We may indicate an unimodality of $f(M)$ for single stars: the peak is within the interval $M \in\left(10^{m}, 12^{m}\right)$. There is an additional peak at $M \in\left(6^{m}, 8^{m}\right)$ for double and triple systems, which is connected with primary components.

However this additional maximum does not appear in the distributions by spectral types (Figure 1, right) and is rather weak in the distributions by color index $B-V$ at $B-V \in(0.5,1.0)$ (see Figure 2, left). The main peaks at spectral types $\mathrm{M}$ and $B-V \in(1.5,2.0)$ are clearer for single stars than for double and triple stars: a contract for $M$ stars versus $K$ stars is about a factor 3.5 in the first case and about a factor 2.5 in the second one.

On average the binaries and triples have a relative deficit of faint compo- 

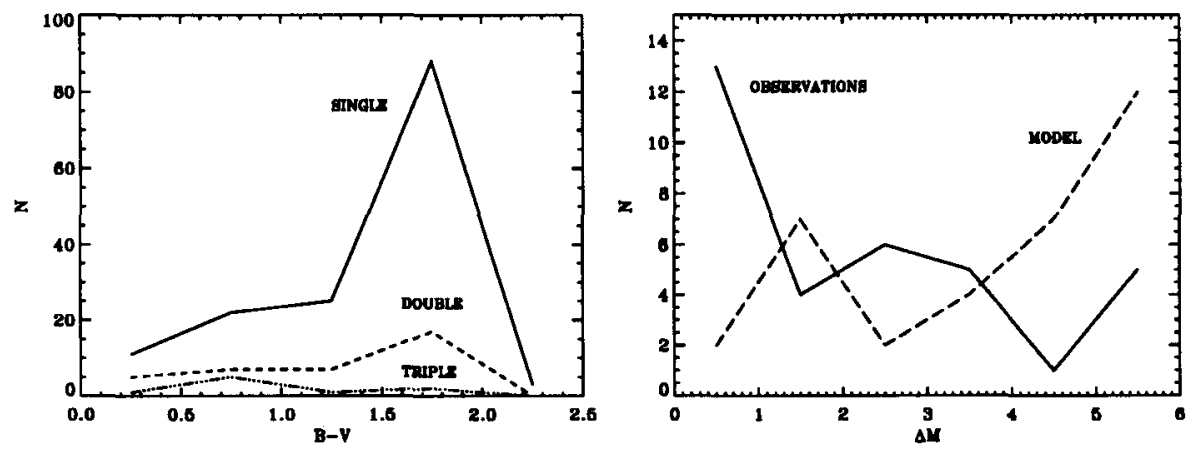

FIGURE 2. (left) The distributions $N(B-V)$ by color index. ((right) The distributions by $\Delta \mathrm{M}$ for the binary components.

nents. This fact may be connected with dynamical evolution of multiple systems when the lightest components may escape from the systems and form a field population for the most part.

\section{CORRELATIONS}

In this section we consider the correlation between the physical properties of components in binaries. For this aim we have constructed the distribution by differences $\Delta M$ of absolute magnitudes of components (Figure 2, right). The observed distribution by $\Delta M$ is shown by solid line. The maximum of this distribution is within $\Delta M \in\left(0^{m}, 1^{m}\right)$.

In order to estimate the statistical significance of this correlation we consider the name sample of 68 components and make a random re-distribution to pairs. The corresponding function $f_{r}(\Delta M)$ is drawn by dashed line in Figure 2, right.

Using the $\chi^{2}$-test we conclude that the actual and randomized distributions by $\Delta M$ are different with a probability $P \geq 0.999$. Therefore there is a significant tendency for correlation between the luminosities of components in double stars, which may be connected with a mechanism of binary formation and/or with a possible evolution of components towards the closeness of luminosities (see, e.g., Kozyrev 1991).

The authors thank very much, Dr. A.S. Rastorguev for the kind possibility to use the preliminary version of Gliese \& Jahreiss Catalogue.

\section{REFERENCES}

Batten, A.H. 1973, Binary and Multiple Systems of Stars, (Oxford, Pergamon Press) Batten, A.H. \& Fletcher J.M. 1989, JRASC, 83, No. 5, 289

Batten, A.H., Fletcher, J.M., \& McCarthy, D.G. 1989, Eighth Catalogue of the Orbital Elements of Spectroscopic Binary Systems, Publ. DAO, XVII

Duquennoy, A. \&Mayor, M. 1991, A\&A, 248, 485

Kozyrev, A.N. 1991, Izbrannye Trudy, Izd. Leningrad University, Leningrad, p. 165 